\title{
Finite Element ANSYS Analysis of the Behavior for 6061-T6 Aluminum Alloy Tubes under Cyclic Bending with External Pressure
}

\author{
Kuo-Long Lee ${ }^{1}$, Chen-Cheng Chung ${ }^{2}$ and Wen-Fung Pan ${ }^{2}$ \\ 1. Department of Innovative Design and Entrepreneurship Management, Far East University, Tainan 70101, Taiwan, R.O.C \\ 2. Department of Engineering Science, National Cheng Kung University, Tainan 70101, Taiwan, R.O.C
}

\begin{abstract}
In this paper, by using adequate stress-strain relationship, mesh elements, boundary conditions and loading conditions, the finite element ANSYS analysis on the behavior of circular tubes subjected to symmetrical cyclic bending with or without external pressure is discussed. The behavior includes the moment-curvature and ovalization-curvature relationships. In addition, the calculated ovalizations at two different sections, middle and right cross-sections, are also included. Experimental data for 6061-T6 aluminum alloy tubes subjected to cyclic bending with or without external pressure were compared with the ANSYS analysis. It has been shown that the analysis of the elastoplatic moment-curvature relationship and the symmetrical, ratcheting and increasing ovalization-curvature relationship is in good agreement with the experimental data.
\end{abstract}

Key words: Cyclic bending, external pressure, moment, curvature, ovalization, finite element ANSYS analysis.

\section{Introduction}

In many engineering applications, such as offshore pipelines, risers, platforms, land-based pipelines, and breeder reactor tubular components are acted upon both cyclic bending and external pressure. It is well known that the ovalization of the tube cross-section is observed when a circular tube is subjected to bending. If the loading history is cyclic bending, the ovalization increases in a ratcheting manner with the number of cycles. However, if the bending is combined with the external pressure, a small amount of external pressure will strongly influence the trend and magnitude of the ovalization. Therefore, the experimental and theoretical studies of the response of circular tubes under cyclic bending combined with external pressure are important for many industrial applications.

Since 1980, Kyriakides and co-workers [1] have conducted experimental and theoretical investigations

Corresponding author: Wen-Fung Pan, Ph.D., professor, research fields: experimental stress analysis, finite element analysis and plasticity. E-mail: z7808034@email.ncku.edu.tw. on the behavior of pipes subjected to bending with or without internal pressure or external pressure. Kyriakides and Shaw [1] performed an experimental investigation on the response and stability of thin-walled tubes subjected to cyclic bending. Corona and Kyriakides [2] investigated the asymmetric collapse modes of pipes under combined bending and external pressure. Kyriakides and Lee [3] experimentally and theoretically investigated the buckle propagation in confined steel tubes. Limam et al. [4] studied the inelastic bending and collapse of tubes in present of the bending and internal pressure. Limam et al. [5] investigated the collapse of dented tubes under combined bending and internal pressure.

Pan and his co-workers [6] also constructed a similar bending machine with a newly invented measurement apparatus, which was designed and set up by Pan et al. [6], to study various kinds of tubes under different cyclic bending conditions. Lee et al. [7] studied the influence of the $D_{o} / t$ (diameter/thickness) ratio on the response and stability of circular tubes 
subjected to cyclic bending. Chang and Pan [8] discussed the buckling life estimation of circular tubes subjected to cyclic bending. Lee et al. [9] investigated the viscoplastic response and collapse of sharp-notched circular tubes subjected to cyclic bending.

Corona and Kyriakides [10] experimentally investigated the response of 6061-T6 aluminum alloy tubes under cyclic bending and external pressure. In their study, the moment-curvature curves revealed a cyclic hardening for 6061-T6 aluminum alloy tube. The moment-curvature curve became steady after a few cycles. In addition, the moment-curvature response exhibits almost no influence by the external pressure. However, the ovalization-curvature behavior increases in a ratcheting symmetrical manner and is strongly influenced by the magnitude of the external pressure. Although Lee et al. [11] used endochronic theory combined with the principle of virtual work to properly simulate the aforementioned behavior, there are several flaws in their theoretical formulation. Firstly, the endochronic theory is too complicated and when it is combined with the principle of virtual work, the numerical method for determining the related parameters becomes extremely difficult. Next, their method treats the same response for every cross section for a circular tube under pure bending. However, based on the experimental data from Corona and Kyriakides [10], the moment and curvature are almost the same for every section, but the ovalization is different for each section. In addition, the response of the 6061-T6 aluminum alloy tube lacks of investigation.

Due to the great progress in computation speed and great improvement in the theory describing the elastoplastic response in finite element method in recent years, the accuracy of calculation by finite element method has become better [4-5, 12-13]. In this study, by considering adequate stress-strain relationships, mesh elements, boundary conditions and loading conditions, the finite element software ANSYS is used to analyze the response of circular tubes subjected to cyclic bending with or without external pressure. Circular tube for 6061-T6 aluminum alloy is considered in this study. The experimental data tested by Corona and Kyriakides [10] are used to compare with the finite element ANSYS analysis. It has been shown that good agreement between the ANSYS analysis and experimental results has been achieved.

\section{Finite Element ANSYS Analysis}

In this study, the finite element software package ANSYS is used for analyzing the behavior of circular tubes subjected to cyclic bending with or without external pressure. The behavior is the relationships among the moment, curvature and ovalization. The elastoplastic stress-strain relationships, mesh element, boundary condition and loading condition of the finite element ANSYS are discussed in the following.

\subsection{Elastoplastic Stress-Strain Relationship}

According to the uniaxial stress-strain curves for 6061-T6 aluminum alloy tested by Corona and Kyriakides [10], the uniaxial stress $(\sigma)$-strain $(\varepsilon)$ curves are constructed in ANSYS as shown in Fig. 1. It can be seen that the curve is constructed by multilinear segments, the number on the curve indicates the order of the segment. In addition, the kinematic hardening rule is used as the hardening rule for cyclic loading.

\subsection{Mesh Element}

Due to the three-dimensional geometry and elastoplastic deformation of the tube, we use the SOLID 185 element for relative analysis. This element is a tetrahedral element built in ANSYS and is suitable for analyzing the plastic or large deformation. In particular, this element is adequate to analyze a shell component under bending. Due to the symmetry of the front and rear, right and left, only one fourth of the tube's model was constructed. Fig. 2 is the mesh of the finite element ANSYS. 


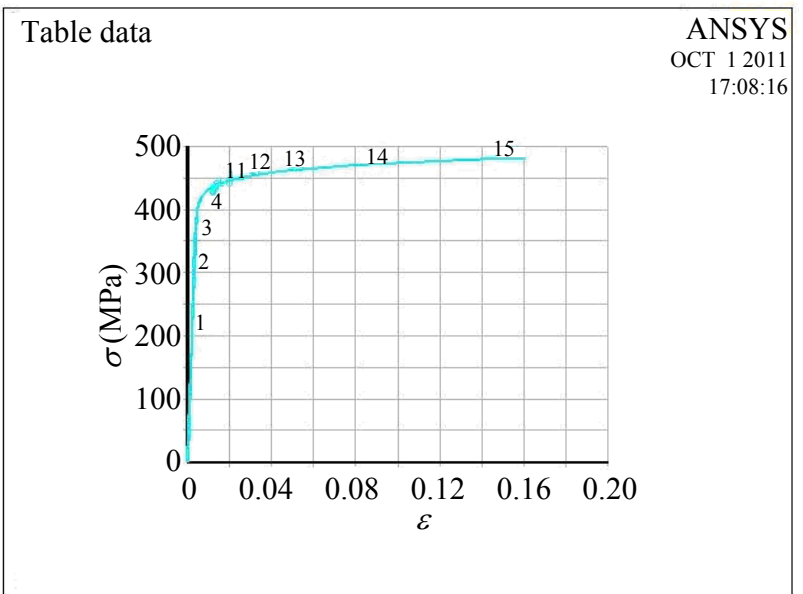

Fig. 1 Uniaxial stress $(\sigma)$-strain $(\varepsilon)$ curve for 6061-T6 aluminum alloy constructed by finite element ANSYS.

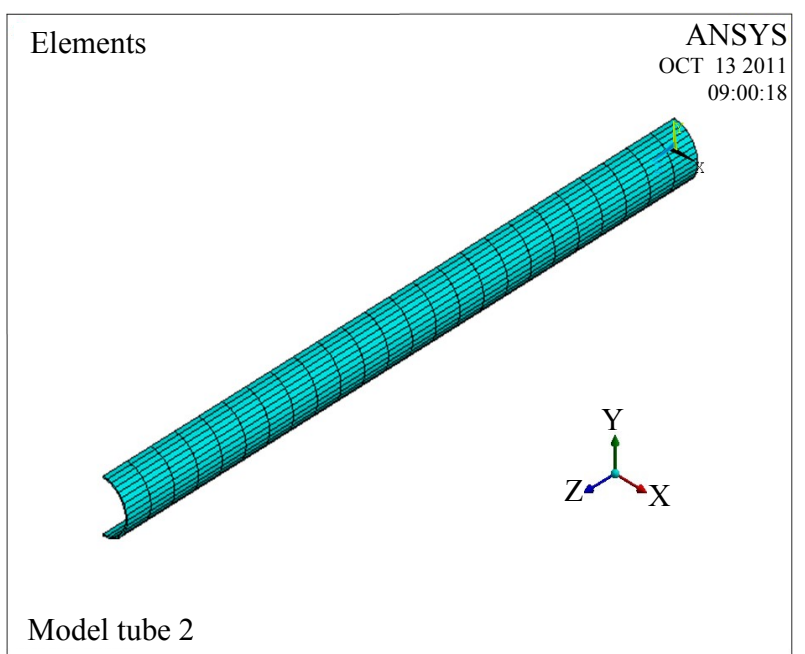

Fig. 2 Mesh constructed by ANSYS.

\subsection{Boundary and Loading Conditions}

Based on the coordinate system of Fig. 2, the pure bending is on the $y$-z plane. The points on the top and bottom of the tube are free to move in $y$-direction and $z$-direction. But they can not move in $x$-direction. Fig. 3 shows the boundary condition of the finite element ANSYS. It can be seen that we use rollers on the top and bottom of the tube to represent the constraints.

In this study, the pure bending is controlled by curvature. The magnitude of the curvature cannot be directly input into ANSYS. Therefore, the corresponding displacements of the points $(1,2, \ldots, N)$ on the center surface (neutral surface) are considered as the input data shown in Fig. 4. The points of the

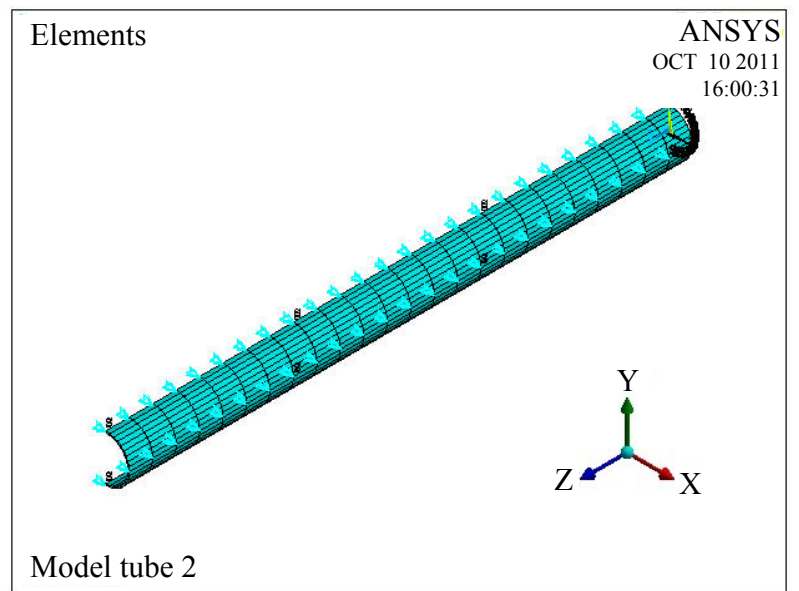

Fig. 3 Boundary conditions constructed by ANSYS.

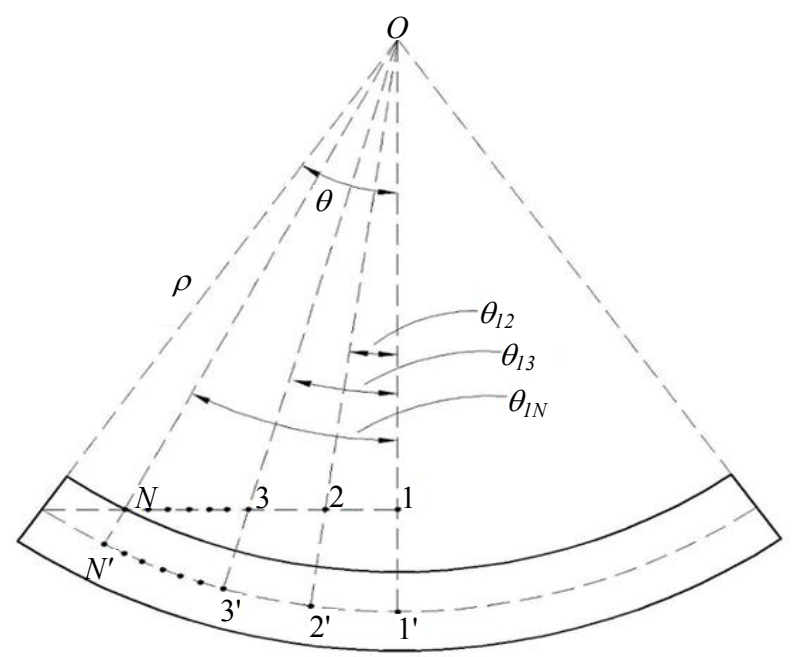

Fig. 4 Loading conditions of the finite element ANSYS.

undeformed center surface are indicated as $1,2, \ldots, N$. Once the tube is subjected to pure bending, the points $1,2, \ldots, N$ move to points $1^{\prime}, 2^{\prime}, \ldots, N^{\prime}$, respectively. For pure bending, the curvature $\kappa$ is:

$$
\kappa=\frac{1}{\rho}=\frac{\theta}{L}
$$

where, $\rho$ is the radius of curvature and $L$ is the half of the original tube's length. Since the loading is curvature-controlled, the magnitudes of $\kappa, \rho$ and $L$ are known quantities. Thus, the magnitude of $\theta$ can be determined from Eq. (1). The vertical displacement of point 1 is:

$$
\left|\overline{11^{\prime}}\right|_{v}=\rho-\left|\overline{O 1^{\prime}}\right|=\rho-\rho \cos \theta
$$

The horizontal displacement of point 1 equals zero. When we consider the displacement of point 2, the 
length $|\overline{12}|$ is a known quantity, the angle of $\theta_{12}$ is determined to be:

$$
\theta_{12}=\tan ^{-1}\left(\frac{|\overline{12}|}{|\overline{O 1}|}\right)=\tan ^{-1}\left(\frac{|\overline{12}|}{\rho \cos \theta}\right)
$$

The length $|\overline{O 2}|$ is found to be

$$
|\overline{O 2}|=\sqrt{|\overline{12}|^{2}+|\overline{O 1}|^{2}}=\sqrt{|\overline{\mid 2}|^{2}+(\rho \cos \theta)^{2}}
$$

The length of $|\overrightarrow{22}|$ is determined as:

$$
\left|\overline{22^{\prime}}\right|=\rho-|\overline{O 2}|
$$

The vertical and horizontal displacements of $|\overline{22}|$ are calculated to be:

$$
\left|\overline{22^{\prime}}\right|_{v}=\left|\overline{22^{\prime}}\right| \cos \theta_{12},\left|\overline{22^{\prime}}\right|_{h}=\left|\overline{22^{\prime}}\right| \sin \theta_{12}
$$

For the displacement of point $N$, the quantities of $\theta_{1 \mathrm{~N}},|\overrightarrow{O N}|,\left|\overrightarrow{N N^{\prime}}\right|$ are determined to be:

$\theta_{1 N}=\tan ^{-1}\left(\frac{|\overline{1 N}|}{\rho \cos \theta}\right),|\overline{O N}|=\sqrt{|\overline{\mid N}|^{2}+(\rho \cos \theta)^{2}}$,

$$
\left|\overrightarrow{N N^{\prime}}\right|=\rho-|\overline{O N}|
$$

The vertical and horizontal displacements of $\left|\overline{N N^{\prime}}\right|$ are calculated to be:

$$
\left|\overline{N N^{\prime}}\right|_{v}=\left|\overline{N N^{\prime}}\right| \cos \theta_{1 N},\left|\overline{N N^{\prime}}\right|_{h}=\left|\overline{N N^{\prime}}\right| \sin \theta_{1 N}
$$

\section{Comparison and Discussion}

In this section, the behavior of 6061-T6 aluminum alloy circular tubes under cyclic bending with or without external pressure tested by Corona and Kyriakides [10] is compared with the finite element ANSYS analysis discussed in Section 2. In their experimental result, the magnitudes of the pressure, moment, and curvature are normalized by the following quantities [10]:

$$
P_{c}=\frac{2 E}{1-v^{2}}\left(\frac{t}{D_{o}}\right)^{3}, M_{o}=3 \sigma_{o} D_{o}^{2} t, \kappa_{l}=\frac{t}{D_{o}^{2}}
$$

where, $E$ is the elastic modulus, $v$ is the Poisson's ratio, $D_{o}$ is the original outside diameter, $t$ is the wall-thickness, and $\sigma_{o}$ is the yield strength. For
6061-T6 aluminum alloy tube, the values of $E, v, D_{o}, t$ and $\sigma_{o}$ are $68.3 \mathrm{GPa}, 0.33,0.03091 \mathrm{~m}, 0.00089 \mathrm{~m}$ and $288 \mathrm{MPa}$, respectively [10].

\subsection{Cyclic Bending without External Pressure}

Fig. 5a presents the experimental result of cyclic moment $\left(M / M_{o}\right)$-curvature $\left(\kappa / \kappa_{l}\right)$ curve for 6061-T6 aluminum alloy tube under curvature-controlled cyclic bending. The external pressure in this case is equal to zero. The $D_{o} / t$ ratio is 34.7 and the cyclic curvature range is from $+0.67 \mathrm{~m}^{-1}$ to $-0.67 \mathrm{~m}^{-1}$. It is observed from the experimental $M / M_{o}-\kappa / \kappa_{l}$ curve that the 6061-T6 aluminum alloy tube shows a steady loop on the first cycle. Fig. 5b shows the corresponding simulated result obtained from ANSYS analysis. It can be seen that there is not any cyclic hardening or

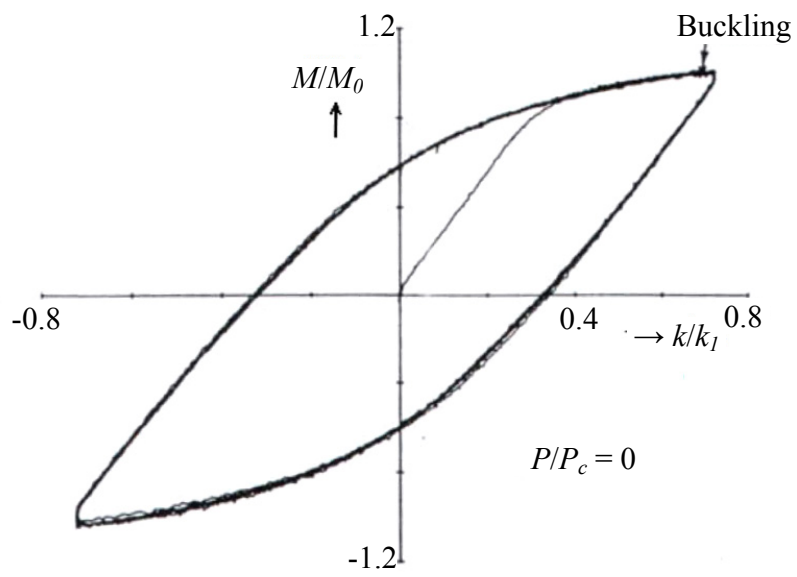

(a) Experiment [10]

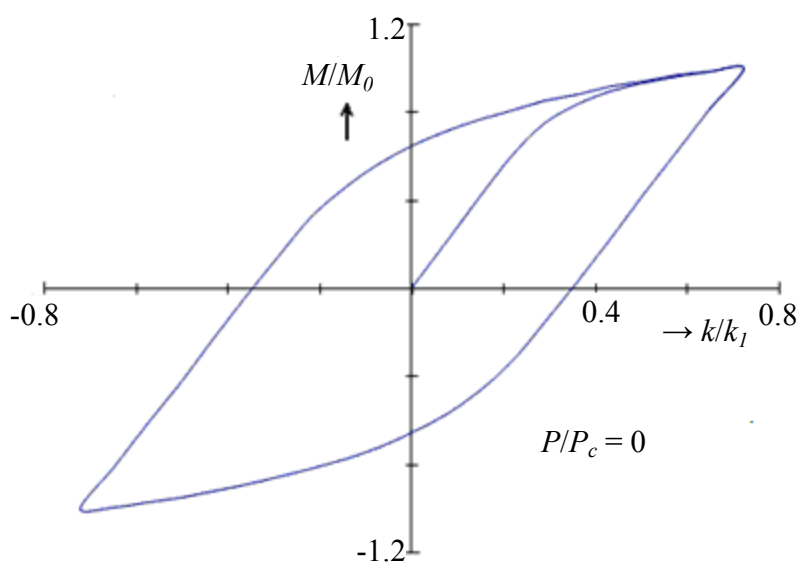

(b) ANSYS analysis

Fig. 5 Experimental and ANSYS analysis moment $\left(M / M_{o}\right)$-curvature $\left(\kappa / \kappa_{l}\right)$ curve for 6061-T6 aluminum alloy tube. 
softening built in ANSYS, thus, only a loop of the $M / M_{o}-\kappa / \kappa_{l}$ curve represents all cyclic bending responses. Fig. 6a depicts the corresponding experimental ovalization of tube cross-section $\left(\Delta D / D_{o}\right)$ as a function of the applied curvature $\left(\kappa / \kappa_{l}\right)$ for Fig. 5a where $\Delta D$ is the change in outside diameter. It can be noted that the ovalization of tube cross-section increases in a symmetrical ratcheting manner with the number of cycles. As the cyclic process continues, the ovalization keeps accumulating. Fig. $6 \mathrm{~b}$ is the corresponding simulated result of $\Delta D / D_{o}-\kappa / \kappa_{l}$ curve.

\subsection{Cyclic Bending with External Pressure}

Fig. 7a presents the experimental result of cyclic

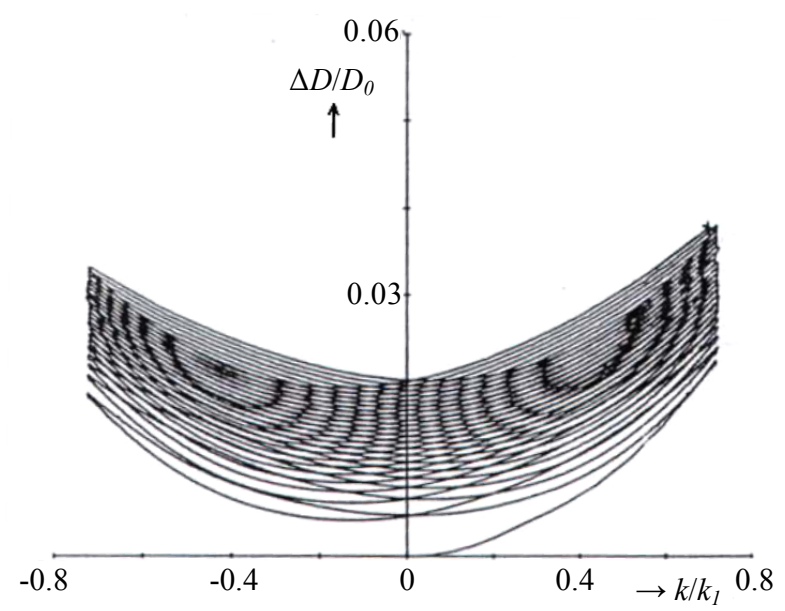

(a) Experiment [10]

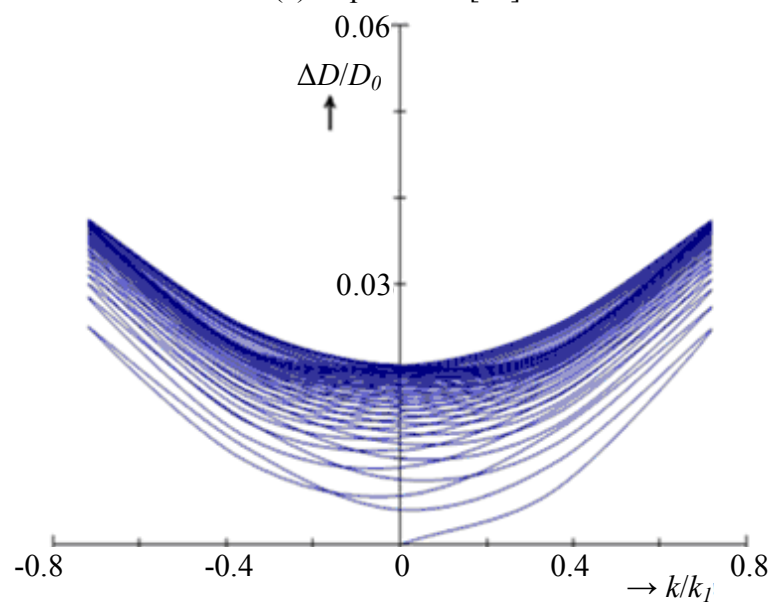

(b) ANSYS analysis

Fig. 6 Experimental and ANSYS analysis ovalization $\left(\Delta D / D_{o}\right)$-curvature $\left(\kappa / \kappa_{l}\right)$ curve for 6061-T6 aluminum alloy tube. moment $\left(M / M_{o}\right)$-curvature $\left(\kappa / \kappa_{l}\right)$ curve for 6061-T6 aluminum alloy tube under cyclic bending with a constant external pressure $P_{c}$ of $1.47 \mathrm{MPa}$. The cyclic curvature range is from $+0.43 \mathrm{~m}^{-1}$ to $-0.43 \mathrm{~m}^{-1}$. Fig. $7 \mathrm{~b}$ demonstrates the corresponding ANSYS analysis result. In their experimental study [10], the length of the tube was around $24 D_{o}$. They measured the ovalization at the position of $11 D_{o}$ indicated as point $\mathrm{A}$ and $18 D_{o}$ indicated as point $\mathrm{B}$ from the right (Fig. 8a). They discovered that the ovalization at point A (shown in Fig. 8a) increases slower than that at point B (shown in Fig. 9a). Figs. $8 \mathrm{~b}$ and $9 \mathrm{~b}$ show the corresponding simulation result of $\Delta D / D_{o}-\kappa / \kappa_{l}$ curve at point $\mathrm{A}$ and $\mathrm{B}$, respectively.

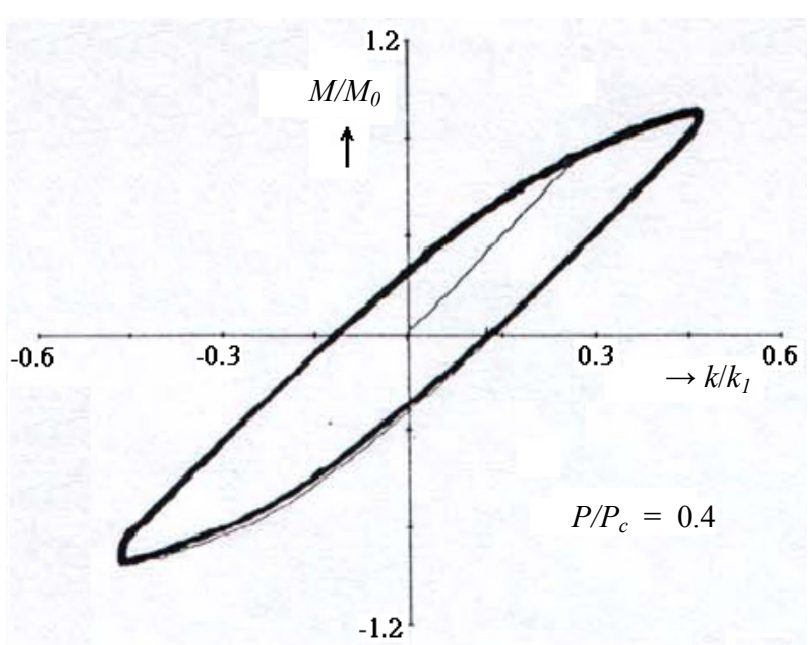

(a) Experiment [10]

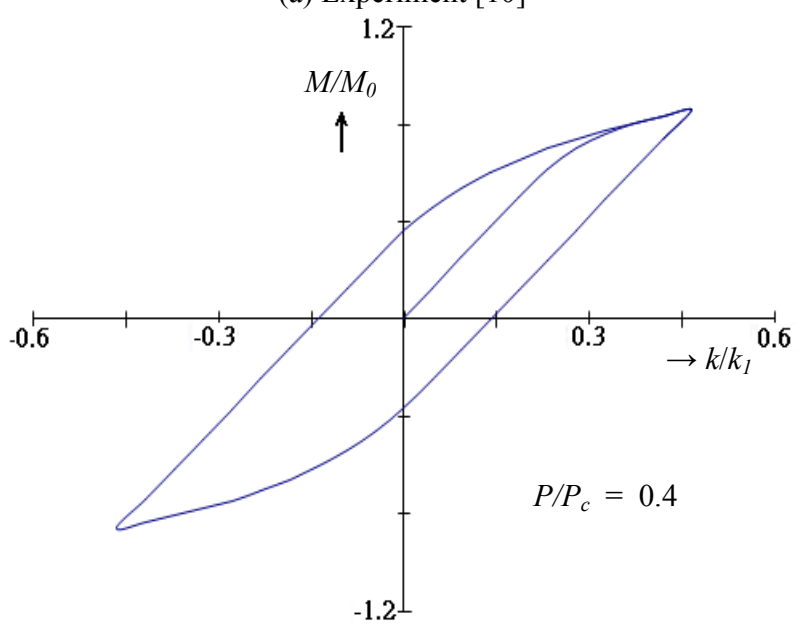

(b) ANSYS analysis

Fig. 7 The experimental result of cyclic moment $\left(M / M_{o}\right)$-curvature $\left(\kappa / \kappa_{l}\right)$ curve for 6061-T6 aluminum alloy tube. 


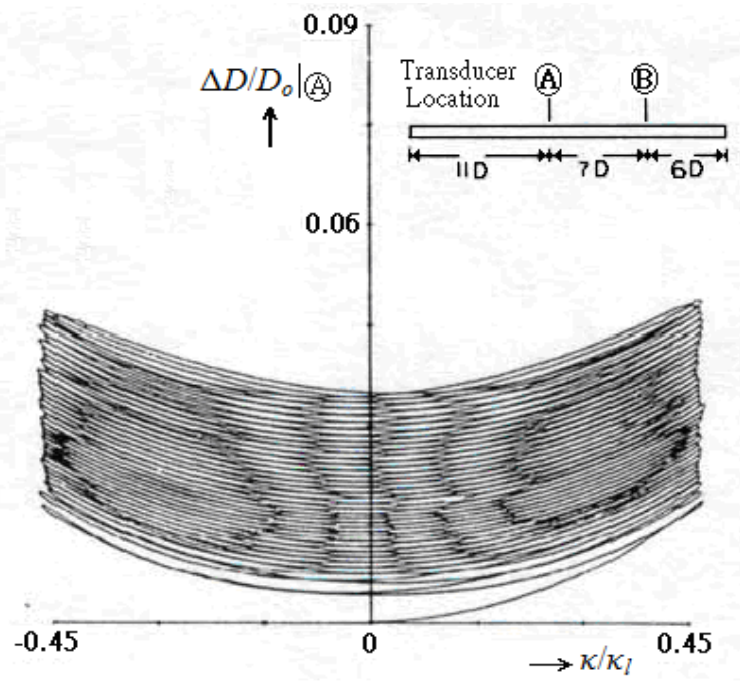

(a) Experiment [10]

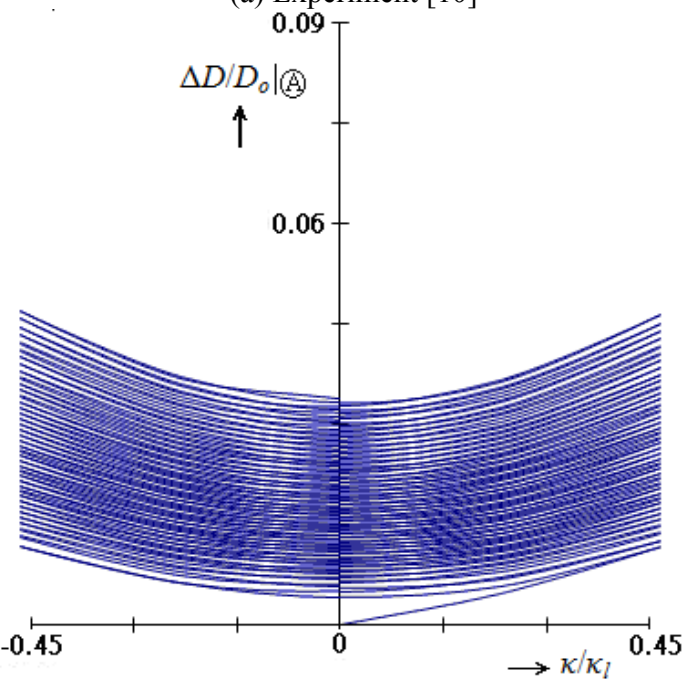

(b) ANSYS analysis

Fig. 8 Experimental and ANSYS analysis ovalization $\left(\Delta D / D_{o}\right)$-curvature $\left(\kappa / \kappa_{l}\right)$ curve at point $A$ for 6061-T6 aluminum alloy tube.

\section{Conclusions}

In this study, the finite element ANSYS with adequate stress-strain relationship, mesh elements, boundary conditions and loading conditions was used to simulate the response of circular tubes subjected cyclic bending with or without external pressure. The experimental data of 6061-T6 aluminum alloy tubes tested by Corona and Kyriakides [10] were used for comparison with the ANSYS analysis. It can be seen that the elastoplatic cyclic loops for moment-curvature

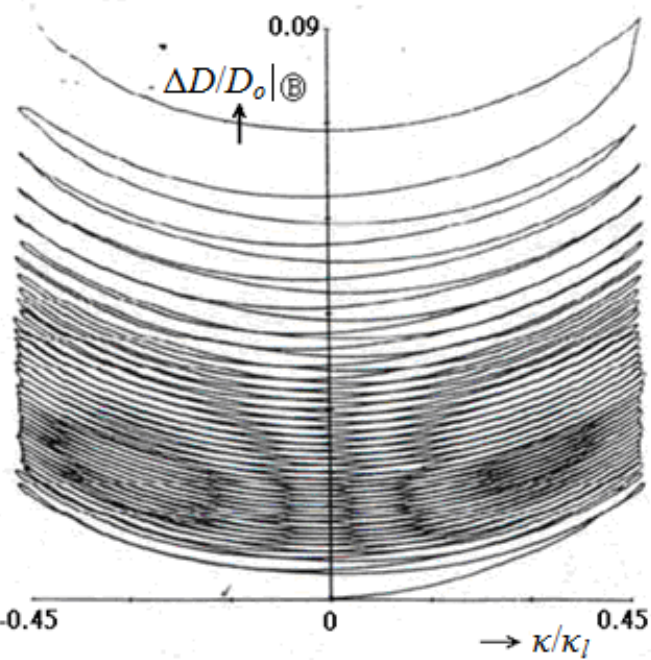

(a) Experiment [10]

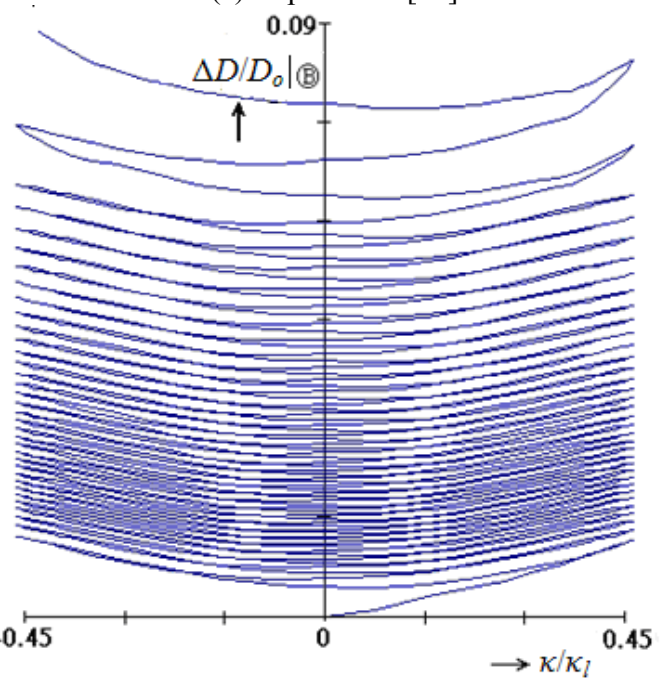

(b) ANSYS analysis

Fig. 9 Experimental and ANSYS analysis ovalization $\left(\Delta D / D_{o}\right)$-curvature $\left(\kappa / \kappa_{l}\right)$ curve at point $B$ for 6061-T6 aluminum alloy tube.

relationship and the symmetrical, ratcheting and increasing ovalization-curvature relationship were properly simulated in Figs. 5b-8b, respectively. In addition, the ovalization at different position can also be well simulated in Fig. 9b.

\section{Acknowledgments}

The work presented was carried out with the support of the National Science Council under grant NSC 100-2221-E-006-081. Its support is gratefully acknowledged. 


\section{References}

[1] S. Kyriakides, P.K. Shaw, Inelastic buckling of tubes under cyclic loads, ASME Journal Pressure Vessels and Technology 109 (1987) 169-178.

[2] E. Corona, S. Kyriakides, Asymmetric collapse modes of pipes under combined bending and external pressure, Journal of Engineering Materials and Technology 126 (12) (2000) 1232-1239.

[3] S. Kyriakides, L.H. Lee, Buckle propagation in confined steel tubes, International Journal of Mechanical Science 47 (2005) 603-620.

[4] A. Limam, L.H. Lee, E. Corana, S. Kyriakides, Inelastic wrinkling and collapse of tubes under combined bending and internal pressure, International Journal of Mechanical Sciences 52 (6) (2010) 37-47.

[5] A. Limam, L.H. Lee, S. Kyriakides, On the collapse of dented tubes under combined bending and internal pressure, International Journal of Solids and Structures 55 (2012) 1-12.

[6] W.F. Pan, T.R. Wang, C.M. Hsu, A curvature-ovalization measurement apparatus for circular tubes under cyclic bending, Experimental Mechanics 38 (2) (1998) 99-102.

[7] K.L. Lee, W.F. Pan, J.N. Kuo, The influence of the diameter-to-thickness ratio on the stability of circular tubes under cyclic bending, International Journal of Solids and Structures 38 (2001) 2401-2413.

[8] K.H. Chang, W.F. Pan, Buckling life estimation of circular tubes under cyclic bending, International Journal of Solids and Structures 46 (2009) 254-270.

[9] K.L. Lee, C.M. Hsu, W.F. Pan, Viscoplastic collapse of sharp-notched circular tubes under cyclic bending, Acta Mech. Solida Sinica 26 (6) (2013) 629- 641.

[10] E. Corona, S. Kyriakides, An experimental investigation degradation and buckling of circular tubes under cyclic bending and external pressure, Thin-Walled Structures 12 (1991) 229-263.

[11] K.L. Lee, C.M. Hsu, C.Y. Hung, Endochronic simulation for the response of 1020 steel tube under symmetric and unsymmetric cyclic bending with or without external pressure, Steel Composite Structures 8 (2) (2008) 99-114.

[12] Y.P. Korkolis, S. Kyriakides, Inflation and burst of anisotropic aluminum tubes for hydroforming applications, International of Plasticity 24 (2008) 509-543.

[13] K.H. Chang, K.L. Lee, W.F. Pan, Buckling failure of 310 stainless steel with different diameter-to-thickness ratios under cyclic bending, Steel and Composite Structures, 10 (3) (2010) 245-260. 